\title{
Thesis
}

\section{Designing awareness systems for social connectedness}

\author{
Thomas Visser \\ Delft University of Technology, The Netherlands
}

\section{Dissertation abstract}

\subsection{Introduction to the topic}

Experiencing social connectedness is important for people's well-being. Hence, people have an intrinsic drive to maintain social connections with their friends and relatives. Computing and communication technology have an increasingly important role in facilitating people in their sense of connectedness over a distance. Telephone, E-mail, and chat technologies have been available to people for more than two decades now, supporting them in actively having conversations amongst each other without physically being together.

Modern communication and information technology makes it possible to communicate more subtle social cues between people. Such social cues are pieces of information that do not contain rich content, such as experiences, thoughts, or ideas. They rather provide a general awareness of presence or action of another person, similar to social cues observed in every day life. For instance, seeing a car parked in front of someone's house, indicating that person is home, or a neighbor waving to say 'hi' without engaging in further conversation. When created unintentionally, these cues are referred to as awareness cues; when created with intention, the cues are called phatic cues. Both types of cues have the potential to support people in having a pervasive sense of social connectedness over a distance, in an addition to communication systems they are already using.

Subtle social cues may be created and displayed through a variety of forms and modalities. This research to explore how interactive everyday products may be used to communicate these cues, and how their presence and use support the user's experience of social connectedness. This ties in to visions on future developments of technology, such as The Invisible Computer, Ambient Intelligence and The Internet of Things, which have described technology to become more and more an integrated part in the fabric of people's everyday lives. Systems primarily designed to support people in communicating subtle cues are referred to as social awareness systems. Although having been studied before, it has remained unclear whether, and how the features of such systems actually contribute to social connectedness, making it problematic for designers to design effective social awareness systems.

The primary goal underlying the research of this dissertation is to generate an increased understanding of how social awareness system should leverage awareness and phatic social cues to support social connectedness for people. The research primarily focuses on the use of tangible forms and modalities, as these are considered to be both more meaningful and better integrated in people's home environment.

\subsection{Studies in this thesis}

The first study explores awareness and phatic cues in an online environment. In a controlled web study, participants received different cues from their confederate partner, being none in the control condition, a randomly timed reminder, an indication of the other person's keystroke activity (awareness), or pokes (phatic cues). Results showed that in this particular case, where partners were unknown to each other, participants did not feel more connected compared to a control condition. However, in this study phatic cues seemed to be less powerful in fostering social connectedness when compared to awareness cues. Since partners were strangers to each other, the effec- 
tiveness of phatic cues may have been low, as participants had no awareness of each other's socialcultural context, making it problematic to attribute meaning to the cues.

Next, an empirical research through design process led to the development of the SnowGlobe research prototype. Five design cases explored the possibilities of tangible interactive concepts combined with a range of communication strategies. The findings were adopted for the design of the lamp SnowGlobe: A social awareness system that enables two users to exchange both awareness and phatic cues between two living rooms, over a distance. As one lamp detects motion in one living room, the other lamp will shine brighter. Additionally, when a user shakes one lamp, the other lamp will blink shortly, and snowflakes will flutter around. The system was designed to be used in field studies for studying the effect of awareness and phatic cues over longer periods of time.

The first field study with the SnowGlobe prototype aimed to understand how people use awareness systems in their daily life, and how it affects their experience of social connectedness. Six user couples used SnowGlobe for at least two weeks each. Based on the results from the questionnaires and interviews conducted in this study, it can be concluded that the system has a positive effect on social connectedness, but it was shown to be problematic to measure how awareness and phatic cues individually contribute to these experiences.

In terms of interaction design, the results confirmed the expected appreciation for a tangible interface. In the domain of social awareness applications, tangible designs may be better able to provide a sense of intimacy, as compared to screen-based systems. The abstract display of SnowGlobe supported users' social connectedness by stimulating them to think of the other person and attribute meaning to the cue. At the same time, it minimized their privacy concerns.

To overcome measurement challenges, a novel field measurement instrument - Closeness Slider was developed. The instrument adopts an Experience Sampling Method strategy, and it was designed to minimize user burden compared to traditional questionnaires. At the same time enabling researchers to prompt field study participants for their current experience of social connectedness, at any time during the field trial. The instrument was validated in a lab study, where it was compared to traditional social connectedness scales. In addition, a pilot field study explored the practical use of the instrument, and helped to fine-tune the protocol for a second field study with SnowGlobe.

The second field study deployed the slider as the primary measurement instrument for understanding how user-system interactions affected the experience of closeness. Four user couples actively used SnowGlobe for three weeks, in which they received daily prompts on the Closeness Slider, partly based on their interaction behavior with SnowGlobe. The Closeness Slider did not collect as much data on particular user-system interaction as expected, but nevertheless, valuable trends were observed. The collected results exposed that sending phatic cues may evoke an increased sense of connectedness. Also, results showed that between participants, responses to various types of cues varied greatly, suggesting that future systems should enable a broad spectrum of subtle communication functionalities.

\subsection{Discussion and design guidelines}

This research has provided insights into the design and perception of subtle social cues through mediated communication. Although the research primarily focused on tangible interactive systems, the insights may also be applicable for nowadays more and more ubiquitous mobile interactive technologies such as tablets and smartphones. Based on the findings from the studies, the following guidelines for the design of social awareness systems were formed:

1. Design how users can create and send awareness and phatic cues. Most design and research work has focused on reception and perception of social cues. The studies in this dissertation have shown the crafting of phatic cues to be as least as important for experiencing social connectedness.

2. Use a spectrum of social interactions, varying in richness, content, and intentionality. People use different strategies in terms of optimizing social connectedness. As a designer, it is therefore key to offer a variety of possible communication functionalities.

3. Consider the use of ambiguity in information, both in sending and receiving, to create engaging social interactions. An ambiguous display stimulates people to actively attribute meaning to the cues they receive, thereby inherently thinking more of the other person. In addition, ambiguity helps to maintain privacy for users.

Social awareness cues, whether in the form of physical products of everyday life, or being an appli- 


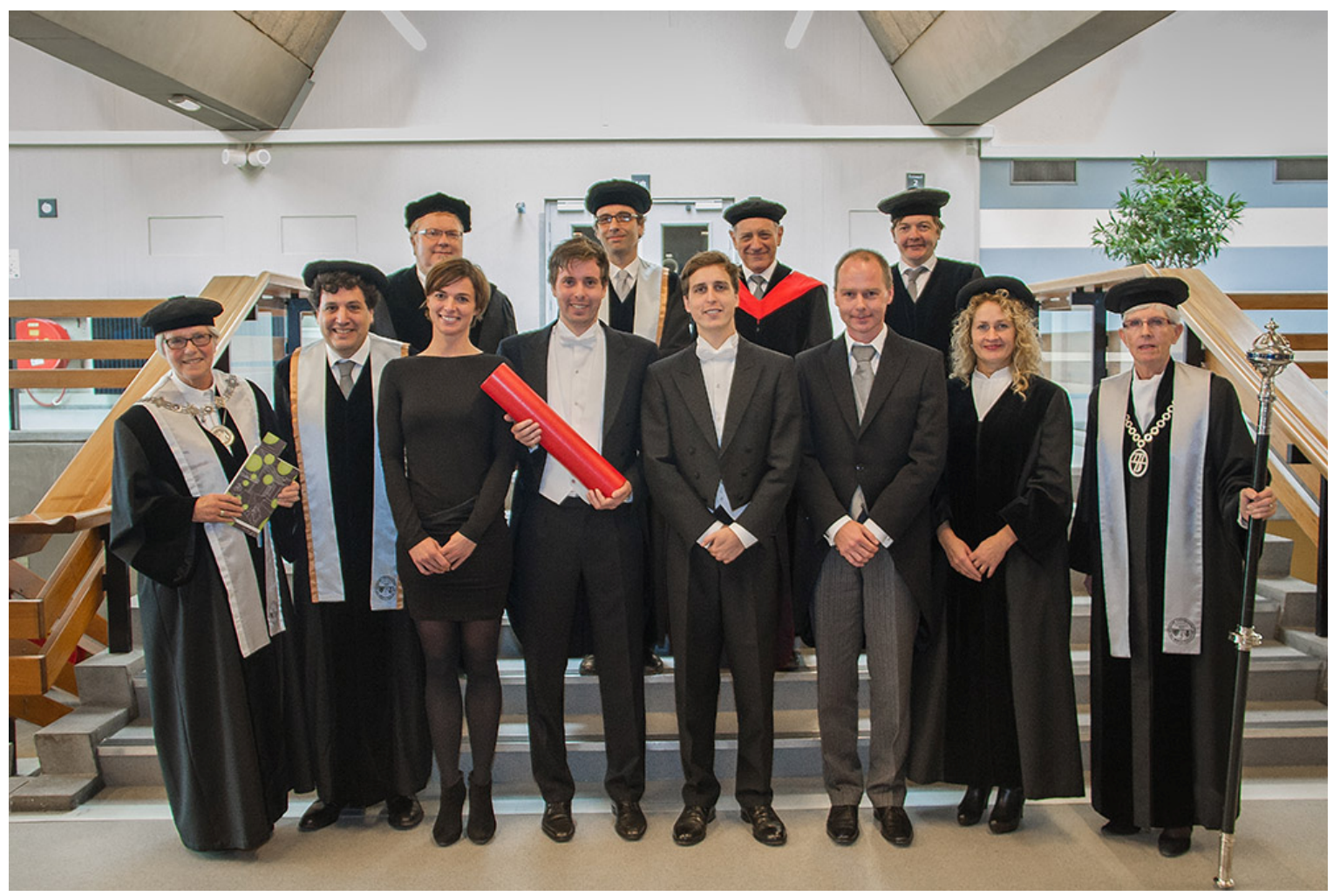

Fig. 1. (Back row from left to right) Prof. Dr. Berry Eggen, Prof. Dr. Paul Hekkert, Prof. Dr. Gerald Cupchik, Prof. Dr. Mark Blythe, (front row from left to right), Prof. Mr. Jitske de Jong, Prof. Dr. David Keyson (supervisor), Nynke Tromp MSc (paranymph), Dr. Thomas Visser (PhD candidate), Sjoerd Visser (paranymph), Dr. Martijn Vastenburg (co-supervisor), and Prof. Dr. Elly Konijn.

cation running on a mobile phone, can increase people's experience of being connected. The guidelines presented in this dissertation aim to support designers in achieving this goal.

\section{PhD defence}

The public defence was held successfully on October 29, 2012, at 10:00am at the Delft University of
Technology. The doctoral committee was formed by the members seen in Fig. 1.

\section{References}

[1] T. Visser, Designing Awareness Systems for Social ConNectedness, 2012, http://repository.tudelft.nl/assets/uuid: a79c4534-6681-4a27-aa1a-e76ccb1c2c35/TVisser_Thesis_s. pdf. 